\title{
Clinical features and outcomes of hepatocellular carcinoma in Caucasian cirrhotic patients on long-term analogue therapy for hepatitis B
}
A. Loglio $^{1}$
M. lavarone ${ }^{1}$
G. Grossi ${ }^{1}$
M. Viganò ${ }^{2}$
MG. Rumi ${ }^{2}$
F. Facchetti ${ }^{1}$
G. Lunghi ${ }^{3}$
A. Sangiovanni ${ }^{1}$
M. Colombo ${ }^{4}$
P. Lampertico ${ }^{1}$ (i)

${ }^{1}$ CRC "A.M. e A. Migliavacca" Center for the Study of Liver Disease, Division of Gastroenterology and Hepatology, Fondazione IRCCS Cà Granda Ospedale Maggiore Policlinico, Università degli Studi di Milano, Milan, Italy

${ }^{2}$ Hepatology Division, Ospedale San Giuseppe, Università degli Studi di Milano, Milan, Italy

${ }^{3}$ Virology Unit, Fondazione IRCCS Cà Granda Ospedale Maggiore Policlinico, Università degli Studi di Milano, Milan, Italy

${ }^{4}$ Center for Translational Hepatology Research, Clinical and Research Center, Humanitas Hospital, Rozzano, Italy

\section{Correspondence}

Dr. P. Lampertico, CRC "A.M. e A. Migliavacca" Center for the Study of Liver Disease, Division of Gastroenterology and Hepatology, Fondazione IRCCS Ca' Granda Ospedale Maggiore Policlinico, Università degli Studi di Milano, Milan, Italy.

Email: pietro.lampertico@unimi.it

Funding information

Italian Ministry of Health, Grant/Award

Number: Ricerca Corrente RC2018/105-01

\section{Summary}

Background: Long-term oral nucleos(t)ide analogue (NUC) therapy in hepatitis B virus (HBV)-related compensated cirrhotics prevents clinical decompensation but not hepatocellular carcinoma (HCC) development.

Aims: To define the clinical features and outcomes of HCC in long-term NUC-treated HBV patients.

Methods: All HCCs developing between 2005 and 2016 in NUC-treated HBV patients under surveillance were studied, excluding those that occurred within the first 6 months of therapy. Clinical features of HCC, alpha faetoprotein (AFP) patterns and patients' outcome were studied.

Results: Seventy-six HCC patients were included. Median age was 67 (40-83) years, 84\% males, 96\% Caucasian, 95\% HBeAg-negative, 96\% with undetectable HBV DNA, 83\% with normal ALT levels, and 92\% with compensated cirrhosis. Median serum AFP levels were 4 (1-3615) $\mathrm{ng} / \mathrm{mL}(>7 \mathrm{ng} / \mathrm{mL}$ in 36\%). HCC was monofocal in $78 \%$, had a median diameter of $20(6-57) \mathrm{mm}$ and was in its early stage in $92 \%$ which allowed potentially curative treatments in 78\% (39\% ablation, 28\% surgical resection, $11 \%$ liver transplantation). Overall, a complete response was obtained in 61 (80\%) patients: in 40 after a first-line treatment, in 3 after the second-line treatment, in 2 after the third-line treatment, while 16 underwent liver transplantation (8 as second line). During 45 (7-144) months after HCC diagnosis, 19 patients died, $84 \%$ from HCC progression. The median time to recurrence was 20.2 (3-53) months, and the cumulative 5 -year liver-related survival was $74 \%$.

Conclusions: HCCs developing in patients under long-term NUC treatment were single, small tumours, amenable to curative therapies able to confer excellent 5-year survival rates. 


\section{1 | INTRODUCTION}

Hepatitis B virus (HBV) is a major health problem worldwide, with roughly 240 million people with chronic infection. ${ }^{1}$ If left untreated, patients with $\mathrm{HBV}$ infection are at high risk of progression to cirrhosis, clinical decompensation, hepatocellular carcinoma (HCC) and liver-related death. ${ }^{2-4}$ International guidelines recommend either a short course of pegylated interferon (Peg-IFN) or the long-term administration of third-generation nucleos(t)ide analogues (NUCs), such as entecavir (ETV) or tenofovir disoproxil fumarate (TDF) or tenofovir alafenamide, to stop the progression of liver disease. ${ }^{2-4}$

Studies with TDF and ETV have clearly shown reversal of clinical decompensation, ${ }^{2}$ prevention of clinical decompensation, as long as $\mathrm{HBV}$ is the only cause of liver damage,,$^{5-9}$ and regression of grade 1 oesophageal varices in most of the patients (83\%) with a negligible risk of de novo occurrence of oesophageal varices. ${ }^{10}$

On the other hand, chemoprevention of HCC by long-term administration of ETV or TDF is still a matter of debate. In ETV-treated patients with cirrhosis, the annual incidence of HCC ranged from $2 \%$ to $4.1 \%$ in Asian studies ${ }^{6,11-13}$ and is $2.6 \%$ in European studies, whereas in European studies of TDF therapy of patients with cirrhosis $\mathrm{HCC}$, risk ranged from $3.7 \%$ to $4 \%,{ }^{8,9,13}$ that is, very similar to the estimates of the course of HBV in untreated patients. ${ }^{14}$ Because the early diagnosis of $\mathrm{HCC}$ increases indications for curative therapies with clear implications on prognosis, identification and close surveillance for the risk of HCC is of strategic importance, ${ }^{2,3}$ and this is also true for ETV or TDF-treated cirrhotic patients. In fact, HCC was the only cause of liver-related death in the 1269 patients without cirrhosis, compared with 503 patients with cirrhosis where $50 \%$ died of liver disease (HCC in $75 \%$ of cases) and $50 \%$ died of nonliver-related causes. ${ }^{15}$ The development of HCC is a major event affecting all causes mortality, whereas it stands as the only factor affecting liver-related mortality in HBV patients under NUCs. Prospective studies with a follow-up long enough to assess the outcome of patients who developed HCC during anti-HBV therapy are lacking.

\section{2 | MATERIAL AND METHODS}

\section{1 | Patients}

All consecutive de novo HCCs diagnosed between 2005 and 31 December 2016 in patients with a HBV-related liver disease on NUC therapy at our outpatient clinic, in 2 different hospitals in Milan-Fondazione IRCCS Cà Granda Ospedale Maggiore Policlinico and Ospedale San Giuseppe, were enrolled in this retrospective study. Exclusion criteria were (1) HCC detected at baseline or occurring within 6 months after starting NUC therapy, (2) HIV and HDV coinfection, as well as autoimmune hepatitis, while co-factors of disease have not been excluded (i.e. alcohol intake, overweight and diabetes). Data on HCC incidence and predictors in NUC-treated patients from our Centres have been previously published. $^{8,10,13,15}$

\section{2 | Measurements}

Routine laboratory analysis and serum HBV DNA were assessed every 3 or 6 months. Serum alanine aminotransferase (ALT) and aspartate aminotransferase were measured with an automated method at $37^{\circ} \mathrm{C}$ (normal value $\leq 40 \mathrm{IU} / \mathrm{L}$ ). Commercially available enzyme immunoassays were used to determine serum antibodies to hepatitis B surface antigen (anti-HBs), hepatitis B e antigen ( $\mathrm{HBeAg}$ ), antibodies to $\mathrm{HBeAg}$, anti-hepatitis Delta (AXSYM, Abbott Laboratories, North Chicago, IL, USA). Anti-hepatitis $C$ virus was assessed by a second-generation enzyme-linked immunoassay (Ortho Diagnostic System, Raritan, NJ, USA). Serum HBV DNA was measured by polymerase chain reaction assay, Cobas Amplicor HBV Monitor Test v2.0 (Roche Diagnostics, Mannheim, Germany) with a lower limit of quantification of $71 \mathrm{IU} / \mathrm{mL}$ from 2005 until 2008 and subsequently by real-time by polymerase chain reaction assay, CobasTaqMan HBV Test v2.0 (Roche Molecular Systems, Inc., Branchburg, NJ, USA) with a lower limit of quantification of $12 \mathrm{lU} / \mathrm{mL}$. HBV genotypes and resistance mutations in the HBV polymerase region were looked for by linear probe assays (INNO-LiPA HBV genotyping and INNO-LiPA HBV DR, Innogenetics NV, Belgium). ${ }^{16}$ Serum AFP levels were determined by ImmunoAssay in Electrochemistry Luminescence "ECLIA" (Roche Diagnostic GmbH, Mannheim, Germany), with a range of $0.605-1210 \mathrm{ng} / \mathrm{mL}$; higher values can be diluted 1:50; normal AFP value $\leq 7 \mathrm{ng} / \mathrm{mL}$.

\section{3 $\mid$ Surveillance and treatment}

The initial anti-HBV treatment was lamivudine or ETV or TDF as monotherapy. For lamivudine-resistant patients, adefovir $10 \mathrm{mg} / \mathrm{d}$ was added from 2003 and switched to TDF $245 \mathrm{mg} / \mathrm{d}$ from 2008. ${ }^{6-8}$ Surveillance for HCC included abdominal ultrasound and AFP every 6 months in cirrhotics, having Child-Pugh Turcotte score A or B, or Child-Pugh Turcotte score $C$ if they could potentially have a liver transplant, according to international recommendations. ${ }^{17}$ Six-month surveillance with AFP monitoring was performed also for patients with advanced fibrosis (Ishak score 4-5). HCC was diagnosed per 2005 American Association for the Study of Liver Diseases criteria until 2010 and thereafter per the updated criteria ${ }^{18,19}$ using contrast imaging techniques like contrast-enhanced ultrasound, computedtomography (CT) scan and magnetic resonance imaging (MRI), whenever the "typical" vascular pattern for HCC of wash-in in the arterial phase and wash-out in the portal or equilibrium phase was demonstrated. An ultrasound-guided fine-needle biopsy was performed to restore sensitivity in nodules escaping radiological diagnosis, using a 21-gauge trenchant needle (Biomol, HS Hospital Service S.P.A., Aprilia, Italy).

At enrolment, all patients underwent staging with abdominal MRI or CT and a chest-CT scan, a bone scintigraphy when clinically required, and were stratified for HCC stage according to Barcelona Clinic Liver Cancer (BCLC) classification. ${ }^{20}$

After HCC diagnosis, all patients were evaluated by a multidisciplinary clinical team composed of a transplant surgeon, a 
hepatologist and an interventional radiologist, with management changing in line with the updating of clinical guidelines when available and according to the specific expertise of each Centre and the general condition of each patient (comorbidities, age) that can modify the stage-related treatment indication, especially in BCLC very early HCC. Moreover, treatment selection also considered other factors such as tumour site. Radio frequency ablation (RFTA) was the first choice for tumour ablation, whereas alcohol injection was a possible alternative for patients with a tumour up to $2 \mathrm{~cm}$. RFTA was contraindicated for strategically localised tumours and for tumours larger than $5 \mathrm{~cm}$ or more than 3 nodules. Patients listed to liver transplantation (LT) were bridged with RFTA or transarterial chemoembolisation (TACE), in any case considering LT as the firstline treatment in the analysis. Response to therapy was defined by EASL and modified RECIST criteria $^{17,21}$ performing a dynamic CT or MRI 1 month after local ablation and each cycle of TACE, 2 months after resection, LT and first administration of sorafenib. Radiological response was evaluated by expert radiologists: complete response was disappearance of any enhancement of the target lesions, partial response was $\geq 30 \%$ decrease of the sum of diameters of viable target lesions, stable disease was any case that did not qualify for previous definitions, and progressive disease was $\geq 20 \%$ increase in the sum of viable target lesions (including emergence of new lesions). Patients achieving a radiological complete response had a CT or MRI repeated every 3 months for 24 months and subsequently were switched to US surveillance at 6-month intervals. Retreatment of patients who failed to achieve a complete response was decided by multidisciplinary clinical team as follows: RFTA was repeated once in patients with incomplete tumour necrosis; TACE was repeated until complete response was achieved, or progression demonstrated. Sorafenib dose was reduced by $50 \%$ in the presence of grade 2 toxicity, whereas it was discontinued whenever tumour progressed or grade 3-4 side-effects did not improve following 7-day discontinuation. ${ }^{22}$ A second-line treatment was proposed by multidisciplinary clinical team for patients with tumour progression, or unfit to further cycles of first-line treatment due to technical constraints, like failure of catheterisation of the feeding artery or ethanol diffusion, residual tumour not suitable to be managed with the first-line treatment, or tumour recurrence. The same policy was adopted for third- and forth-line treatments. Patients were followed until death or last available clinical evaluation, up to 31 July 2017. Patients who missed a scheduled visit were contacted by phone call to collect information on clinical status and outcome.

\section{4 | Definitions}

Cirrhosis was diagnosed by histology or on clinical grounds by using an abdominal ultrasound features of blunted, nodular liver edge accompanied by splenomegaly $(>13 \mathrm{~cm})$ and $<100 \times 10^{3} / \mu \mathrm{L}$ platelets. Persistent HBV suppression meant HBV DNA persistently under the lower limit of quantification of the test used throughout the follow-up.
Rescue transplant was offered to all patients failing first-line therapy for $\mathrm{HCC}$ or with recurrence after complete response to treatment with the following conditions: extension of the tumour according to the Milan criteria and age less than 65 years up to 2013 and less than 70 years later.

\section{5 | Study endpoints}

The primary endpoints of the study were clinical features of HCC and alpha-fetoprotein patterns. Secondary endpoints were the response to treatments, development of early and late recurrence after therapy for HCC, and survival.

\subsection{Statistical analysis}

Data were expressed as counts and percentages for qualitative variables and as median and range for continuous discrete variables. Significance of differences in the distribution of quantitative and qualitative variables was assessed with Student's $t$ test, KruskalWallis, Fisher's exact or chi-square tests as appropriate. All $P$ values were 2-tailed and a level of 0.05 was considered statistically significant. The Kaplan-Meier method was used to estimate outcome rates. The log-rank test was used to compare curves between patient groups. The cumulative incidence of HCC was estimated by competing risks framework, with death and liver transplantation as potential competing events, by using the nonparametric KalbfleischPrentice method. Statistical analysis was performed with STATA (Stata Statistical Software: Release 7.0. College Station, TX: Stata Corporation).

\section{3 | RESULTS}

The study population included 76 subjects after the exclusion of one patient with a HCC developed after liver transplantation, one with a tumour detected outside regular surveillance and 2 patients with a tumour detected within the first 6 months of NUC therapy.

The demographical and clinical features of the enrolled patients are depicted in Table 1: at the time of HCC, 73 (96\%) patients had undetectable HBV DNA, 72 (95\%) were HBeAg negative, and 63 (83\%) had normal ALT levels. All patients were under anti-HBV treatment: 57 (75\%) with third-generation NUC (58\% on TDF, 17\% on ETV), 10 (13\%) on lamivudine monotherapy and 9 (12\%) on lamivudine + adefovir. Anti-HBV therapy started 81 (6-190) months before HCC development. Seventy (92\%) patients had cirrhosis, all with a compensated disease; the remaining 6 patients had advanced fibrosis (Ishak S4/5). In these patients, no difference in number of co-factors of HCC development has been identified compared with the cirrhotic patients of the cohort (data not shown).

Despite NUC treatment, an ongoing hepatic necroinflammation was present in 13 patients: elevated ALT levels were associated with alcoholic abuse and older age at multivariate analysis, but not with overweight or coinfection (data not shown). Only 3 subjects tested 
TABLE 1 Demographical, clinical and virological characteristics of the 76 patients with HBV-related hepatocellular carcinoma developed during long-term treatment with nucleos(t)ide analogues

\begin{tabular}{|c|c|}
\hline Variable & $N=76$ \\
\hline Age, $y^{a}$ & $67(40-83)$ \\
\hline Males & 64 (84\%) \\
\hline \multicolumn{2}{|l|}{ Ethnicity } \\
\hline Caucasian & 73 (96\%) \\
\hline Asian & $2(3 \%)$ \\
\hline Indian Americans & $1(1 \%)$ \\
\hline Family history of hepatocellular carcinoma & $2(3 \%)$ \\
\hline Alcohol abuse & $3(4 \%)$ \\
\hline Smoking habits & $19(25 \%)$ \\
\hline Overweight (BMI 25-29.9 kg/m²) & 29 (38\%) \\
\hline Obesity $\left(\mathrm{BMI}>30 \mathrm{~kg} / \mathrm{m}^{2}\right)$ & $10(13 \%)$ \\
\hline Class I & 9 \\
\hline Class II & 1 \\
\hline Diabetes & $12(16 \%)$ \\
\hline Cirrhosis $^{\mathrm{b}}$ & 70 (92\%) \\
\hline \multicolumn{2}{|l|}{ Child-Pugh Turcotte score } \\
\hline A & 64 (91\%) \\
\hline B & $5(8 \%)$ \\
\hline C & $1(1 \%)$ \\
\hline Transient elastography value $>12 \mathrm{kPa}^{\mathrm{c}}$ & 15 (38\%) \\
\hline Oesophageal varices & $13(17 \%)$ \\
\hline Small-sized varices & $10(13 \%)$ \\
\hline Medium/large-sized varices & $3(4 \%)$ \\
\hline $\mathrm{qHBsAg}, \mathrm{IU} / \mathrm{mL}^{\mathrm{d}}$ & $644(2-10650)$ \\
\hline $\mathrm{HBeAg}$ negative & $72(95 \%)$ \\
\hline HBV DNA undetectable & $73(96 \%)$ \\
\hline Genotype $D^{\mathrm{e}}$ & $40(89 \%)$ \\
\hline \multicolumn{2}{|l|}{ Nucleos(t)ide analogues } \\
\hline ETV or TDF \pm Lamivudine & $57(75 \%)$ \\
\hline Lamivudine & $10(13 \%)$ \\
\hline Lamivudine+Adefovir & $9(12 \%)$ \\
\hline Duration of Nucleos(t)ide analogues treatment, $\mathrm{mo}^{\mathrm{a}}$ & $81(6-190)$ \\
\hline ALT $<41 \mathrm{IU} / \mathrm{L}$ & $63(83 \%)$ \\
\hline
\end{tabular}

BMI, body mass index; ETV, entecavir; TDF, tenofovir; ALT, alanine aminotransferase; $\mathrm{qHBsAg}$, quantitative hepatitis $\mathrm{B}$ surface antigen; $\mathrm{HBeAg}$, hepatitis $\mathrm{B}$ e antigen.

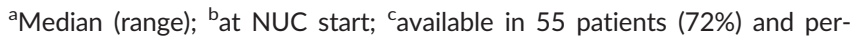
formed within 6 mo before diagnosis; davailable in 41 patients (54\%); eavailable in 45 patients (59\%) at NUC start. Obesity grade I: BMI $30-$ $34.9 \mathrm{~kg} / \mathrm{m}^{2}$; obesity grade II: $\mathrm{BMI} \geq 35 \mathrm{~kg} / \mathrm{m}^{2}$.

positive for HCV RNA: their HCC features and hepatitis activity were comparable to HBV monoinfected NUC-treated subjects. Both transaminases and alpha-fetoprotein levels were within the normal range for all these $3 \mathrm{HCV} \mathrm{HBV} \mathrm{cirrhotic} \mathrm{patients,} \mathrm{as} \mathrm{well} \mathrm{as} \mathrm{median}$ age, BMI, portal hypertension features and platelets count were similar to HBV monoinfected group.
HCC was radiologically diagnosed in 65 (86\%) patients and histologically in the remaining 11 (14\%): 59 patients (78\%) had a single tumour node, and the median tumour size was $20 \mathrm{~mm}$ (range: 6-57), AFP $\leq 7 \mathrm{ng} / \mathrm{mL}$ in $64 \%$ of patients, within "Milan criteria" in 71 (93\%) resulting in BCLC stage O/A in 70 (92\%) (Table 2). Among the 5 patients with a more advanced $\mathrm{HCC}$ stage, no difference in number of co-factors of HCC aggressiveness has been identified compared with those patients with earlier stages (data not shown).

\section{1 | Treatment algorithm}

The first-line therapy was any potentially curative treatment in 59 (78\%) patients: 30 (59\%) were treated by RFTA, 21 (41\%) by surgical resection (including the 6 patients without cirrhosis) and 8 patients were listed for LT as first treatment approach. In these patients listed for LT, 1 patient was staged BCLC D for liver decompensation, while 7 patients were excluded from other potentially curative treatments due to severe portal hypertension or site of tumour localisation. RFTA was the bridge therapy in 2 patients and TACE or radioembolisation in additional 3 patients, while 3 patients directly underwent LT.

Seventeen patients $(22 \%)$ were first treated by non curative approaches: TACE in 13 (76\%), radioembolisation in 2 (12\%) and systemic medical treatment in 2 (12\%). Twelve BCLC 0/A (17\%) staged patients were, therefore, treated by TACE.

Overall, excluding the 8 patients receiving LT as first treatment, 40 (59\%) patients obtained a complete response after the first treatment, 13 (19\%) patients had a partial response, 2 (3\%) patients treated with sorafenib had a stable disease, and $13(19 \%)$ patients had a disease progression after the first treatment. Among the 40 patients

TABLE 2 Characteristics of tumours of the 76 patients with HBV-related hepatocellular carcinoma developed during long-term treatment with nucleos(t)ide analogues

\begin{tabular}{|c|c|}
\hline Variable & $N=76$ \\
\hline Single tumour node & 59 (78\%) \\
\hline Node size, $\mathrm{mm}^{\mathrm{a}}$ & $20(6-57)$ \\
\hline In "Milan criteria" & 71 (93\%) \\
\hline In "Up to 7 criteria" & 71 (93\%) \\
\hline Extra-hepatic disease $^{\mathrm{b}}$ & $2(3 \%)$ \\
\hline \multicolumn{2}{|l|}{ BCLC staging system } \\
\hline 0 & 17 (22\%) \\
\hline A & $53(70 \%)$ \\
\hline B & $2(3 \%)$ \\
\hline C & $3(4 \%)$ \\
\hline $\mathrm{D}$ & $1(1 \%)$ \\
\hline AFP levels, $\mathrm{ng} / \mathrm{mL}^{\mathrm{a}}$ & $4(1-3615)$ \\
\hline$>7$ & 27 (36\%) \\
\hline$>200$ & $4(5 \%)$ \\
\hline
\end{tabular}

BCLC, Barcelona Clinic for Liver Cancer; AFP, alpha-fetoprotein.

${ }^{\mathrm{a}}$ Median (range); ${ }^{\mathrm{b}}$ Macrovascular portal vein invasion in 2 and lymphnode metastasis in 1 . 
with a complete response after the first treatment, 24 (60\%) patients maintained complete response during 45.4 (5-144) months of followup, while 16 (40\%) patients experienced recurrence in 20.2 (3-53) months (Figure 1). The recurrences were treated by surgical resection (1 patient), RFTA (5 patients), TACE (8 patients) and sorafenib (2 patients).

Among the 13 patients with partial response to first-line treatment, the second-line treatment consisted in LT enlisting in 5 patients, while 7 patients repeated RFTA, and 1 patient repeated TACE.

Overall, a complete response was obtained in 45 (58\%) patients: in 40 after a first-line treatment, in 3 after the second-line treatment, and in 2 after a third-line treatment.

Among the 13 patients who experienced disease progression after the first-line treatment, 3 patients were enlisted for LT, 2 repeated RFTA, 3 TACE ( 2 repeated and 1 progression after RFTA), 2 sorafenib (both progressions after RFTA) and 3 received best supportive care only.

Overall, 16 patients received liver transplants: 8 as first line and 8 as a rescue after partial response or progression. In our case series, among the 16 transplanted patients, HCC recurred in 1 case (6\%) 8 months after LT with lung and liver localisation (LT had been performed as a rescue treatment after partial response to radioembolisation for a BCLC B staged HCC).

Fifteen (20\%) patients received a HCC treatment outside the BCLC treatment algorithm: 8 BCLC 0 ( 5 treated by TACE due to strategic localisation of the lesions and 3 underwent LT) and 7 BCLC A were treated by TACE due to localisation of the lesions (4), comorbidities (3) and multifocality (1). Among the 12 BCLC $0 / A$ patients treated by TACE, complete response was obtained in $6(50 \%)$ patients.

\section{2 | Survival}

During 45 (range 2-144) months after HCC diagnosis, 19 (25\%) patients died: 15 (79\%) due to HCC progression and 4 for extra- hepatic reasons (one after myocardial infarction, one for cerebral haemorrhage, one for pancreatic cancer and one for lung cancer progression). No patient died for end-stage liver disease. Among the 15 deaths due to HCC progression: 11/70 BCLC 0/A, 1/2 BCLC B and 2/3 BCLC C. Along the same period, 5 (7\%) patients were lost (all $\mathrm{BCLC}$ 0/A).

The median overall survival was 45 (7-144) months, corresponding to a 5-year overall survival of $69 \%$ (95\% Cl 54\%-79\%) and a 5year overall survival censoring patients when transplanted of $62 \%$ (95\% Cl 46\%-75\%) (Figure 2). Considering only the 70 patients staged BCLC 0/A, the median overall survival was 47 (7-144) months, corresponding to a 5 -year overall survival of $73 \%(95 \% \mathrm{Cl}$ $59 \%-84 \%$ ), and a 5 -year overall survival of $67 \%$ (95\% Cl 50\%-79\%) when transplanted patients were censored. Finally, the median liverrelated survival was 45 (7-144) months, corresponding to a 5-year overall survival $74 \%$ (95\% Cl 60\%-84\%), and a 5-year overall survival censoring patients when transplanted of $68 \%$ (95\% Cl 51\%-80\%). The 5-year overall survival limited to the 16 transplanted patients was $93 \%$ : only 1 patient died for progression of an $\mathrm{HCC}$ recurrence 8 months after LT.

\section{DISCUSSION}

In this study, the vast majority of the HCC identified during surveillance of long-term NUC treated compensated Caucasian cirrhotic patients were small nodules within "Milan criteria" amenable to curative approaches. To the best of our knowledge, this is the first large cohort study reporting survival benefits in patients with a HCC developed during NUC therapy, with approximately $70 \%$ of the patients alive after 5 years, without deaths attributable to liver decompensation.

The present study provides several new findings. First, the clinical and biological features of HCC occurring in long-term

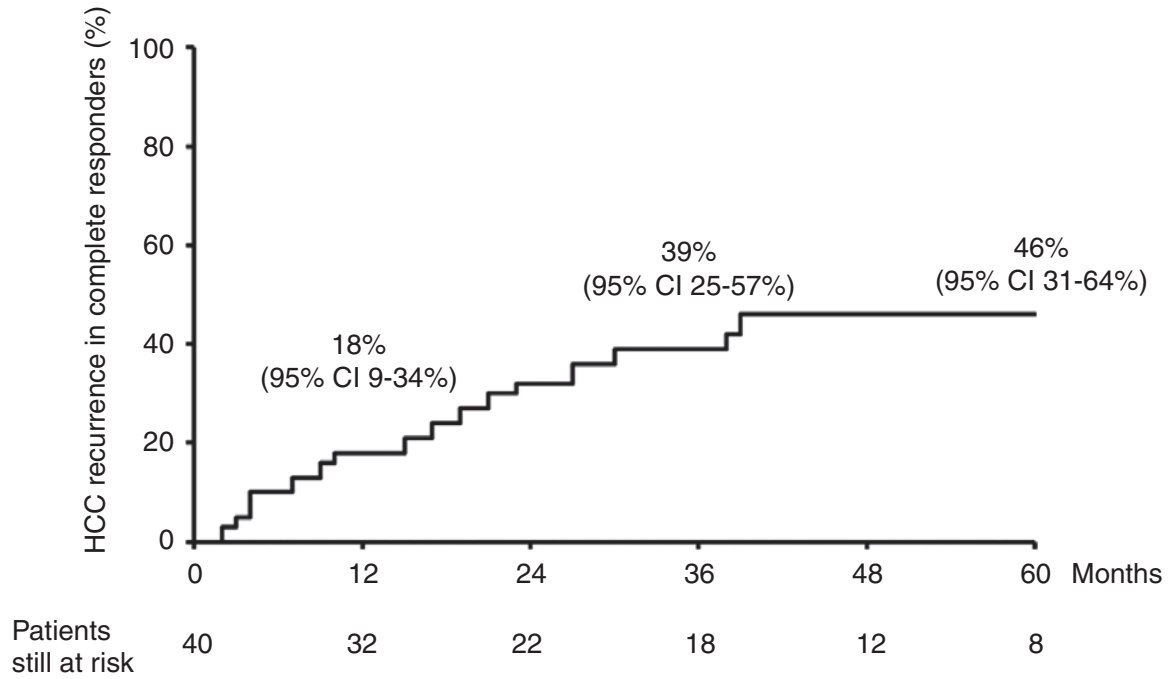

FIGURE 1 Time to hepatocellular carcinoma recurrence in 40 patients who had a complete radiological response after first-line treatment. Excluded were 8 patients who underwent liver transplantation as a first-line treatment 


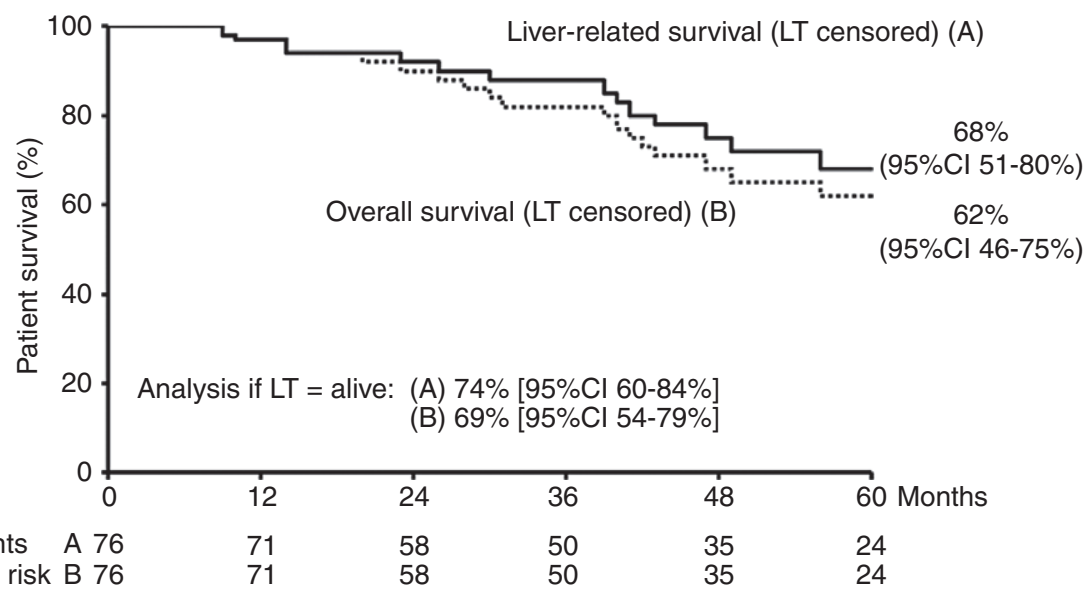

FIGURE 2 Cumulative 5-year overall and liver-related survival in 76 patients with hepatocellular carcinoma included in the study

NUC-treated patients compare favourably with previous studies in untreated cirrhotics and in NUC-treated patients from different geographical areas. Several studies have previously shown that HBVrelated $\mathrm{HCC}$ are aggressive tumours in significant proportion of patients, ${ }^{23}$ but these studies were generated before oral therapy with new potent NUC for HBV became widely available. This indeed is not what has been found in our patients who, in the vast majority of cases, presented with early, small, single HCC - as high as $92 \%$, with $93 \%$ of our patients within "Milan criteria" - assuring the best treatment options (curative treatment options offered to $78 \%$ of our patients) and the best prognosis. This is an unprecedented finding for Caucasian cirrhotic patients as the only other similar, but smaller in size, studies came from Korea and Hong-Kong. ${ }^{24-26}$ There are several possible explanations for this finding. Compliance to $\mathrm{HCC}$ surveillance was optimised by recall policies and frequent visits to the Liver centre due to the need of NUC dispensation, coupled with a dedicated multidisciplinary team in an academic setting. Moreover, in our country, HCC surveillance is free of charge for patients with advanced chronic liver disease and this may make the access to surveillance easier. However, besides logistics, biological factors might have also have accounted for the good prognosis of HCC patients under NUC. It has been previously shown that long-term effective anti-HBV oral therapy reduces inflammation and fibrosis in the liver by means of modification of adaptive immune reactions developing because of chronic HBV infection. The effective control of HBV DNA replication leads to a release reduction of cytokines and of growth factors, thus reducing the necrosis of hepatocytes and proliferation of fibroblasts. Furthermore, lowering the high turnover of hepatocytes characteristic of active hepatitis reduces the risk of host DNA mutations, responsible for their malignant transformation. $^{27-30}$ The finding of a limited number of HCC detected in advanced stages was more likely due to the limited sensibility of ultrasounds in excluding HCC (14\%-20\% in large studies) than the presence of patients at higher risk. ${ }^{31}$ Moreover, no demographical, clinical and virological risk factors appeared associated in different survival outcomes or in HCC recurrence rates out of 40 patients with complete response after first-line treatment.
The fact that controlled studies and meta-analysis demonstrated that long-term oral NUC therapy reduces the incidence of HCC provides some support to this hypothesis, while prolonged NUC therapy fails to cancel HCC risk, since it is unable to act on integrated viral sequences and covalently closed circular HBV DNA, both factors contributing to chromosomal instability, to activation of cancerrelated genes and to inactivation of protective genes. ${ }^{27-30}$

The most important finding of the study was the excellent 5-year survival of patients developing HCC while on long-term NUC therapy for HBV. This important clinical finding compares favourably with previous studies in untreated or NUC-treated patients from Asia. ${ }^{7,14,32-34}$ Different studies in Asia, comparing untreated patients with patients treated with NUC, showed a better overall survival and liver-related survival in treated patients, even when a statistical significance was not reached in consequence of small sample size or short follow-up of the studies. ${ }^{32,33}$ These data were confirmed by 2 meta-analyses focusing on NUC impact in patients with $\mathrm{HCC}$ treated by curative treatments (ie surgery or ablative procedures) and noncurative treatments (TACE and sorafenib), respectively. ${ }^{7,34}$ All included studies were from Asia, frequently small in sample size and retrospective in study design. Some studies were performed with firstgeneration NUC. ${ }^{7,34}$ However, by pooling the data from the studies reporting overall survival, the results showed a significantly lower risk of death among the NUC-treated group. ${ }^{7,34}$

Focusing on the treated groups of these Asian studies, the 5-year survival of patients with HCC developed during treatment with NUC varied between $16 \%$ and $40 \%$, according to the stage and treatments included in the different studies. ${ }^{7,32-34}$ The difference of overall survival in their studies compared with ours might be due do the following: more advanced stage of HCC, no access to liver transplantation, less patients achieving maintained viral suppression, and therefore a residual risk of liver decompensation. Many of these studies included patients treated with NUC only after HCC treatments, and frequently only when a complete response had been obtained, which is a different study design than our present study. The prolonged survival in patients with long-term NUC treatment could be partially justified by the lack of the competing risk of death 
due to concurrent liver disease progression. Indeed, recent long-term cohort studies in Caucasian HBV patients with long-term treatment of ETV or TDF have clearly demonstrated that the risk of HCC-free clinical decompensation is minimal and that HCC is the leading cause of liver-related deaths. ${ }^{8,15}$ In our study, the 5-year overall survival was $69 \%$, independently of patients' stage, treatment modality and $\mathrm{HCC}$ recurrence.

To our knowledge, this is the first report to date to show these survival rates in Caucasian HCC patients with a prolonged follow-up. Previous studies were limited to Taiwan showing a 2-year survival rate of $80 \%$ and 6-year survival rate of $67 \%$ in $\mathrm{HCC}$ treated with RFTA and surgical resection, respectively. ${ }^{32,33}$ These studies have limited impact in our clinical practice, since NUC was started after HCC treatment and interrupted after a predefined period of time. As previously demonstrated in the PAGE study, the 5-year survival rate of Caucasian HBV patients treated with ETV/TDF can be excellent, with a significant proportion of deaths coming from unrelated liver causes and HCC development being a major factor affecting the overall mortality and the only factor affecting liver-related mortality in such patients. The same was true in 2 studies from FarEast. $^{13,15,35-37}$ In our cohort-limited to patients with HCC -we have a proportion of deaths attributable to other causes, while all liver-related deaths were due to HCC progression, with no death attributable to liver decompensation. This is probably the strongest evidence of NUC impacting on the course of HBV-related HCC. Another possible explanation for this finding is the strict adherence to surveillance protocol in our cohort, granting the identification of small treatable tumours. Prolonged suppression of HBV infection might have played a major role in ensuring the best possible clinical outcomes, because multiple anti-tumour procedures could have been offered over time to patients with well-preserved liver function, ensuring the best possible management of HCC. Given the favourable efficacy/safety ratio of NUC therapies and their widespread use in clinical practice, we presume that this advantage will progressively improve. Moreover, our study showed a low risk of recurrence in NUC-treated patients with complete response after any HCC treatment: only $39 \%$ of patients experienced HCC recurrence after 3 years. Our data confirmed previous findings in Taiwanese patients after RFTA and surgical resection, with a recurrence rate of $47 \%$ and $25 \%$ at 2 years, respectively, in NUC-treated patients. ${ }^{7,34}$ Several studies have reported that NUC therapy can reduce the risk of HCC development, ${ }^{11,12,35-37}$ both in Western and Eastern cohorts. Here, we made a further step forward, demonstrating that NUC therapy can be associated to a low risk of HCC recurrence among patients with HBV-related HCC after any treatment able to provide a complete response. HCC itself may have had a relatively indolent course in long-term NUC-treated patients, due to reduction of inflammatory milieu of liver parenchyma surrounding the tumour. Late recurrence of HCC after curative treatments has been shown to be related to the background of liver disease, such as hepatic inflammation and liver damage. Moreover, the increased expression of genes involved in inflammatory responses by cells surrounding the tumour indicated a field effect, in which environmental exposure-as active viral replication-lead to an increased potential for future malignant transformation. ${ }^{37}$ The absence of viral replication in our patients may have reduced this risk accordingly.

A separate analysis should be made on the 16 transplanted patients of our cohort, attaining a 5-year overall survival of $93 \%$ and a recurrence rate of $6 \%$. This survival was particularly prolonged not only compared to historical studies on liver transplants for HCC, but also when compared to recent data from the European Liver Transplant Registry on HCC in HBV patients as primary indication for liver transplantation (5-year survival 75\%). ${ }^{38}$ This is probably due to both the prevention of post-LT HBV-reinfection and the limited indication to LT within "Milan criteria" in almost all patients, therefore with lower tumour burden and decreased risk of recurrence, as we have previously showed in a larger cohort of liver transplanted patients for HCC in NUC therapy. ${ }^{39}$

Finally, the diagnostic accuracy of AFP levels was limited as only $36 \%$ of the patients had serum levels above $7 \mathrm{ng} / \mathrm{mL}$, only $20 \%$ above $20 \mathrm{ng} / \mathrm{mL}$, and $5 \%$ above $200 \mathrm{ng} / \mathrm{mL}$. These findings are in line with other data previously published in a similar subset of patients in Korea, ${ }^{25}$ where patients with current or recent exposure to NUC therapy showed poorer performance of AFP compared with untreated patients, speculating that HBV replication might directly induce AFP expression in HCC cells while NUC therapy suppresses expression of AFP in HCC, leading to decreased sensitivity of AFP. Contrasting to these and our findings, 2 other studies in Asia in patients treated long-term with NUC therapy showed high positive predictive value for HCC development if AFP levels persistently or abruptly elevated more than 12 or even $6 \mathrm{ng} / \mathrm{mL}^{25,26}$ All studies, in agreement with our findings, showed a high specificity of AFP increase during NUC therapy as long as the ALT levels were normal.

There are a few limitations in this study, especially the lack of an untreated control group. However, after the pivotal trial on lamivudine in 1997 showing the clinical benefits of NUC therapy in patients with advanced $\mathrm{HBV},{ }^{40}$ it is unethical to keep cirrhotic patients untreated, at least in high-income countries. On the other hand, it would be hard to compare these data to those of historical cohorts, due to significant progresses achieved in surgical and locoregional treatments in the last decades. A second limitation is the sample size, even though this is to date the largest cohort for the analysis of HCC management and survival in Caucasian NUCtreated patients. Third, the study was conducted in a tertiary referral, academic centre, where a large expertise in the management of these patients accumulated over the last 30 years. Finally, our patients were homogeneous in terms of geographical origin, ethnicity, HBV genotype, and disease severity, characteristics that may limit the applicability of our finding in other regions. At the same time, the study has several strengths. It describes the largest cohort study to date, patients were managed according to criteria agreed upon internationally, the endpoint was as strong as survival could be, and patients were homogenous and managed by a single-centre unit.

In conclusion, this study demonstrates that the vast majority of HCC developing in Caucasian compensated cirrhotic patients long- 
term NUC treated are small, Milan-in, BCLC 0/A lesions amenable of potentially curative treatments with substantial survival benefits. While acknowledging that these data must be confirmed in other independent cohorts, this study sheds new lights on a relevant topic given that $\mathrm{HCC}$ is almost the only complication observed in patients with HBV permanently suppressed by NUC.

\section{ACKNOWLEDGEMENTS}

The study is partially supported by Italian Ministry of Health ("Ricerca Corrente 2018"). We thank Megan Zinn for English revision and Roberta Ferrari for the editorial assistance.

Declaration of personal interests: Massimo lavarone has served as a speaker for Bayer, Gilead Science, Janssen, BTG, Abbvie, and as a consultant for BTG. Mauro Viganò has served as a speaker for Roche, Gilead Sciences, BMS. Angelo Sangiovanni has served as a speaker for Bayer, Gilead Science, Janssen, BTG, Abbvie, Novartis, and an advisory board member for Tiziana science. Massimo Colombo has served as a speaker for Tibotec, Roche, Novartis, Bayer, BMS, Gilead Sciences, Vertex, Merk, Janssen, Abbvie, and an advisory board member for Merk, Roche, Novartis, Bayer, BMS, Gilead Sciences, Tibotec, Vertex, Janssen Cilag, Achillion, Lundbeck, GSK, GenSpera, AbbVie, Alfa Wasserman, and has received funding from BMS, Gilead Sciences. Pietro Lampertico has served as a speaker and an advisory board member for BMS, Roche, Gilead Sciences, GSK, MSD and Abbvie. Others: none.

Declaration of funding interests: This study was funded by Italian Ministry of Health, grant "Ricerca Corrente RC2018/105-01".

\section{AUTHORSHIP}

Guarantor of the article: P. Lampertico.

Author contributions: M. lavarone and P. Lampertico, concept and design of the study; A. Loglio, M. Viganò and G. Grossi, data collection; F. Facchetti and G. Lunghi, laboratory analyses; A. Loglio, A. Sangiovanni and P. Lampertico, statistical analysis; M. lavarone, A. Loglio and P. Lampertico, writing of article; MG. Rumi and M. Colombo, final revision and study supervision; all authors approved the final version of the manuscript.

\section{ORCID}

A. Loglio (iD http://orcid.org/0000-0001-8022-8803

M. lavarone (iD http://orcid.org/0000-0003-3493-6504

MG. Rumi iD http://orcid.org/0000-0002-5876-8528

G. Lunghi iD http://orcid.org/0000-0003-1579-3149

P. Lampertico iD http://orcid.org/0000-0002-1026-7476

\section{REFERENCES}

1. Stasi C, Silvestri C, Voller F, Cipriani F. The epidemiological changes of HCV and HBV infections in the era of new antiviral therapies and the anti-HBV vaccine. J Infect Public Health. 2016;9:389395.
2. European Association for the Study of the Liver. EASL 2017 Clinical Practice Guidelines on the management of hepatitis B virus infection. J Hepatol. 2017;67:370-398.

3. Terrault NA, Bzowej NH, Chang KM, et al. AASLD guidelines for treatment of chronic hepatitis B. Hepatology. 2016;63:261-283.

4. Sarin SK, Kumar M, Lau GK, et al. Asian-Pacific clinical practice guidelines on the management of hepatitis B: a 2015 update. Hepatol Int. 2016;10:1-98.

5. Wong GL, Chan HL, Mak CH, et al. Entecavir treatment reduces hepatic events and deaths in chronic hepatitis B patients with liver cirrhosis. Hepatology. 2013;58:1537-1547.

6. Zoutendijk R, Reijnders JG, Zoulim F, et al. Virological response to entecavir is associated with a better clinical outcome in chronic hepatitis B patients with cirrhosis. Gut. 2013;62:760-765.

7. Lim YS, Han S, Heo NY, et al. Mortality, liver transplantation, and hepatocellular carcinoma among patients with chronic hepatitis B treated with entecavir vs lamivudine. Gastroenterology. 2014;147:152-161

8. Papatheodoridis GV, Dalekos GN, Yurdaydin C, et al. Incidence and predictors of hepatocellular carcinoma in Caucasian chronic hepatitis B patients receiving entecavir or tenofovir. J Hepatol. 2015;62:363-370.

9. Lampertico P, Soffredini R, Yurdaydin C, et al. Four years of tenofovir monotherapy for NUC naïve field practice European patients suppresses HBV replication in most patients with a favorable renal safety profile but does not prevent HCC in patients with or without cirrhosis. Hepatology. 2013;58:A933.

10. Lampertico $P$, Invernizzi $F$, Viganò $M$, et al. The long-term benefits of nucleos $(\mathrm{t})$ ide analogs in compensated HBV cirrhotic patients with no or small esophageal varices: a 12-year prospective cohort study. J Hepatol. 2015;63:1118-1125.

11. Hosaka T, Suzuki F, Kobayashi M, et al. Long-term entecavir treatment reduces hepatocellular carcinoma incidence in patients with hepatitis B virus infection. Hepatology. 2013;58:98-107.

12. Wong GL, Chan HL, Chan HY, et al. Accuracy of risk scores for patients with chronic hepatitis B receiving entecavir treatment. Gastroenterology. 2013;144:933-944.

13. Papatheodoridis G, Dalekos G, Sypsa V, et al. PAGE-B predicts the risk of developing hepatocellular carcinoma in Caucasians with chronic hepatitis B on 5-year antiviral therapy. J Hepatol. 2016;64:800-806.

14. Raffetti E, Fattovich G, Donato F. Incidence of hepatocellular carcinoma in untreated subjects with chronic hepatitis B: a systematic review and meta-analysis. Liver Int. 2016;36:1239-1251.

15. Papatheodoridis G, Dalekos G, Yurdaydin C, et al. Excellent 5-year survival in caucasian chronic hepatitis $\mathrm{B}(\mathrm{CHB})$ patients with or without cirrhosis under long-term entecavir (ETV) or tenofovir (TDF) therapy and the impact of hepatocellular carcinoma (HCC). J Hepatol. 2015;62:S263-S264.

16. Stuyver L, van Geyt C, De Gendt S, et al. Line probe assay for monitoring drug resistance in hepatitis B virus-infected patients during antiviral therapy. J Clin Microbiol. 2000;38:702-707.

17. Bruix J, Sherman M, Llovet JM, et al. Clinical management of hepatocellular carcinoma. Conclusions of the Barcelona-2000 EASL conference. European Association for the Study of the Liver. J Hepatol. 2001;35:421-430.

18. Bruix J, Sherman M. Management of hepatocellular carcinoma. Hepatology. 2005;42:1208-1236.

19. Bruix J, Sherman M. American Association for the Study of Liver Diseases. Management of hepatocellular carcinoma: an update. Hepatology. 2011;53:1020-1022.

20. Bruix J, Reig M, Sherman M. Evidence-based diagnosis, staging, and treatment of patients with hepatocellular carcinoma. Gastroenterology. 2016;150:835-853.

21. Lencioni R, Llovet JM. Modified RECIST (mRECIST) assessment for hepatocellular carcinoma. Semin Liver Dis. 2010;30:52-60. 
22. lavarone M, Cabibbo G, Piscaglia F, et al. Field-practice study of sorafenib therapy for hepatocellular carcinoma: a prospective multicenter study in Italy. Hepatology. 2011;54:2055-2063.

23. Ou DP, Yang LY, Huang GW, Tao YM, Ding X, Chang ZG. Clinical analysis of the risk factors for recurrence of $\mathrm{HCC}$ and its relationship with HBV. World J Gastroenterol. 2005;11:2061-2066.

24. Chung JW, Kim BH, Lee CS, et al. Optimizing surveillance performance of alpha-fetoprotein by selection of proper target population in chronic hepatitis B. PLoS ONE. 2016;11:e0168189.

25. Wong GL, Chan HL, Tse YK, et al. On-treatment alpha-fetoprotein is a specific tumor marker for hepatocellular carcinoma in patients with chronic hepatitis B receiving entecavir. Hepatology. 2014;59:986-995.

26. Shim JJ, Kim JW, Lee CK, Jang JY, Kim BH. Oral antiviral therapy improves the diagnostic accuracy of alpha-fetoprotein levels in patients with chronic hepatitis B. J Gastroenterol Hepatol. 2014;29:1699-1705

27. Neuveut C, Wei Y, Buendia MA. Mechanisms of HBV-related hepatocarcinogenesis. J Hepatol. 2010;52:594-604.

28. Tan YJ. Hepatitis B virus infection and the risk of hepatocellular carcinoma. World J Gastroenterol. 2011;17:4853-4857.

29. Guerrieri F, Belloni L, Pediconi N, Levrero M. Molecular mechanisms of HBV-associated hepatocarcinogenesis. Semin Liver Dis. 2013;33:147-156.

30. Budhu A, Wang XW. The role of cytokines in hepatocellular carcinoma. J Leukoc Biol. 2006;80:1197-1213.

31. Chong CC, Wong GL, Wong VW, et al. Antiviral therapy improves post-hepatectomy survival in patients with hepatitis B virus-related hepatocellular carcinoma: a prospective-retrospective study. Aliment Pharmacol Ther. 2015;41:199-208.

32. Lee TY, Lin JT, Zeng YS, Chen YJ, Wu MS, Wu CY. Association between nucleos(t)ide analog and tumor recurrence in hepatitis $B$ virus-related hepatocellular carcinoma after radiofrequency ablation. Hepatology. 2016;63:1517-1527.

33. Wong JS, Wong GL, Tsoi KK, et al. Meta-analysis: the efficacy of anti-viral therapy in prevention of recurrence after curative treatment of chronic hepatitis B-related hepatocellular carcinoma. Aliment Pharmacol Ther. 2011;33:1104-1112.

34. Wu C-Y, Chen Y-J, Ho HJ, et al. Association between nucleoside analogues and risk of hepatitis $\mathrm{B}$ virus-related hepatocellular carcinoma recurrence following liver resection. J Amer Med Assoc. 2012;308:1906.

35. Su TH, Hu TH, Chen CY, et al. Four-year entecavir therapy reduces hepatocellular carcinoma, cirrhotic events, and mortality in chronic hepatitis B patients. Liver Int. 2016;36:1755-1764.

36. Papatheodoridis GV, Chan HL-Y, Hansen BE, Janssen HL, Lampertico P. Risk of hepatocellular carcinoma in chronic hepatitis B: assessment and modification with current antiviral therapy. J Hepatol. 2015;62:956-967.

37. Hoshida Y, Villanueva A, Kobayashi M, et al. Gene expression in fixed tissues and outcome in hepatocellular carcinoma. N Engl J Med. 2008;359:1995-2004.

38. Burra P, Germani G, Adam R, et al. Liver transplantation for HBVrelated cirrhosis in Europe: an ELTR study on evolution and outcomes. J Hepatol. 2013;58:287-296.

39. Viganò $M$, Bhoori $S$, Lampertico $P$, et al. Extended survival of patients with persistently suppressed hepatitis B transplanted for hepatocellular carcinoma. Liver Int. 2015;35:2187-2193.

40. Lai CL, Chien RN, Leung NW, et al. A one-year trial of lamivudine for chronic hepatitis B. Asia Hepatitis Lamivudine Study Group. N Engl J Med. 1998;339:61-68.

How to cite this article: Loglio A, lavarone M, Grossi G, et al. Clinical features and outcomes of hepatocellular carcinoma in Caucasian cirrhotic patients on long-term analogue therapy for hepatitis B. Aliment Pharmacol Ther. 2018;00:1-9. https://doi.org/10.1111/apt.14848 